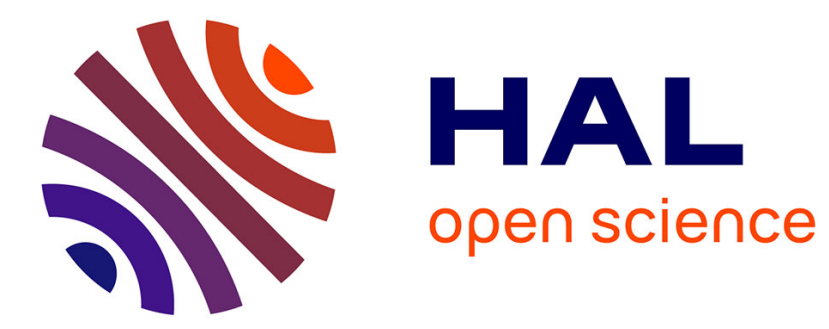

\title{
A concise derivation of membrane theory from three-dimensional nonlinear elasticity
}

David Steigmann

\section{To cite this version:}

David Steigmann. A concise derivation of membrane theory from three-dimensional nonlinear elasticity. Journal of Elasticity, 2009, 97 (1), pp.97-101. hal-00773265

\section{HAL Id: hal-00773265 \\ https://hal.science/hal-00773265}

Submitted on 12 Jan 2013

HAL is a multi-disciplinary open access archive for the deposit and dissemination of scientific research documents, whether they are published or not. The documents may come from teaching and research institutions in France or abroad, or from public or private research centers.
L'archive ouverte pluridisciplinaire HAL, est destinée au dépôt et à la diffusion de documents scientifiques de niveau recherche, publiés ou non, émanant des établissements d'enseignement et de recherche français ou étrangers, des laboratoires publics ou privés. 


\title{
A concise derivation of membrane theory from three-dimensional nonlinear elasticity
}

\author{
David J. Steigmann \\ Department of Mechanical Engineering \\ University of California \\ Berkeley, CA. 94720
}

Abstract: Membrane theory may be regarded as a special case of the Cosserat theory of elastic surfaces, or, alternatively, derived from three-dimensional elasticity theory via asymptotic or variational methods. Here we obtain membrane theory directly from the local equations and boundary conditions of the three-dimensional theory.

\section{Three-dimensional prismatic bodies}

Let $\mathbf{F}$, with $\operatorname{det} \mathbf{F}>0$, be the gradient of a deformation function $\boldsymbol{\chi}(\mathbf{X})$, where $\mathbf{X}$ is the position of a material point in a reference placement. Let $\hat{\mathbf{P}}(\mathbf{F})$ be a smooth constitutive function for the Piola stress $\mathbf{P}$ in an elastic body, and let $W(\mathbf{F})$ be a twice-differentiable function furnishing the strain energy stored in the body, per unit reference volume. Then, $\hat{\mathbf{P}}(\mathbf{F})=W_{\mathbf{F}}$, the derivative with respect to the deformation gradient. To simplify matters we suppose the material to be uniform in the sense that the functions $\hat{\mathbf{P}}$ and $W$ do not depend explicitly on reference position.

We consider deformations that are $C^{2}$ in the interior of the body and we suppress body forces. If such a deformation is equilibrated, then the associated stress satisfies

$$
\operatorname{Div} \mathbf{P}=\mathbf{0}
$$

pointwise, where Div is the divergence with respect to position in the reference placement. We assume that equilibria satisfy the strong-ellipticity condition

$$
\mathbf{a} \otimes \mathbf{b} \cdot \mathcal{M}(\mathbf{F})[\mathbf{a} \otimes \mathbf{b}]>0 \quad \text { for all } \mathbf{a} \otimes \mathbf{b} \neq \mathbf{0},
$$

where

$$
\mathcal{M}(\mathbf{F})=W_{\mathbf{F F}}
$$

is the tensor of elastic moduli.

We note that the constitutive hypothesis of strong ellipticity is used in [1] to prove an existence theorem for $C^{2}$ equilibria under restrictive conditions on the boundary data. Our assumption of strong ellipticity at equilibrium, without restrictions on the boundary data, is insufficient to meet the hypotheses of this theorem. It is nevertheless consistent with the degree of smoothness assumed for the equilibrium deformation, and so it is natural to restrict attention to deformations that are sufficiently smooth for (1) to be meaningful everywhere in the interior of the body. The relaxation of this restriction in the context of the theory of gamma convergence is known to yield a model that is energetically optimal 
$[2,3]$ relative to the present model. However, in contrast to the procedure developed here, the method of gamma convergence does not extend to dynamical problems.

For purposes of illustration we take the reference placement to be the prismatic region $\Omega \times[-h / 2, h / 2]$, where $\Omega$ is a plane and $h$ is the thickness of the body. Let $\mathbf{k}$ be a unit vector that orients the plane, and let

$$
\mathbf{1}=\mathbf{I}-\mathbf{k} \otimes \mathbf{k}
$$

where $\mathbf{I}$ is the identity for three-space. Then,

$$
\mathbf{P}=\mathbf{P} \mathbf{1}+\mathbf{P k} \otimes \mathbf{k}
$$

Further, let $\varsigma$ be a linear coordinate in the direction of $\mathbf{k}$, and suppose $\varsigma=0$ on $\Omega$. Equation (1) is then equivalent to

$$
\operatorname{div}(\mathbf{P} \mathbf{1})+\mathbf{P}^{\prime} \mathbf{k}=\mathbf{0}
$$

where $(\cdot)^{\prime}=\partial(\cdot) / \partial \varsigma$ and div is the two-dimensional divergence with respect to position on $\Omega$. This holds at all points in the interior of the body. In particular,

$$
\operatorname{div}\left(\mathbf{P}_{0} \mathbf{1}\right)+\mathbf{P}_{0}^{\prime} \mathbf{k}=\mathbf{0}
$$

where the subscript ${ }_{0}$ identifies the values of functions at $\varsigma=0$; i.e., on the plane $\Omega$. Thus,

$$
\mathbf{P}_{0}=\hat{\mathbf{P}}\left(\mathbf{F}_{0}\right)
$$

and

$$
\mathbf{P}_{0}^{\prime} \mathbf{k}=\left\{\mathcal{M}\left(\mathbf{F}_{0}\right)\left[\mathbf{F}_{0}^{\prime}\right]\right\} \mathbf{k}
$$

Using the projection (4), we also derive [4]

$$
\mathbf{F}_{0}=\nabla \mathbf{r}+\mathbf{d} \otimes \mathbf{k} \quad \text { and } \quad \mathbf{F}_{0}^{\prime}=\nabla \mathbf{d}+\mathbf{g} \otimes \mathbf{k}
$$

where $\mathbf{r}, \mathbf{d}$ and $\mathbf{g}$ are the restrictions to $\Omega$ of $\chi, \chi^{\prime}$ and $\chi^{\prime \prime}$, respectively, and $\nabla$ is the two-dimensional gradient on $\Omega$.

\section{Membrane equations}

The foregoing equations, holding on $\Omega$, are exact consequences of the three-dimensional theory. Approximations arise in using them to represent material response in $\Omega \times[-h / 2, h / 2]$. We are interested in the leading-order model for small thickness. The latter is assumed to be much smaller than any other length scale in the problem at hand, and the smallest of these is used as the unit of length, so that $h \ll 1$ when non-dimensionalized. In particular, lateral traction data at $\varsigma= \pm h / 2$ impose restrictions on the fields $\mathbf{d}$ and $\mathbf{g}$ arising in the leading-order model for small thickness. Let $\mathbf{t}^{ \pm}$be the Piola tractions applied at the lateral surfaces of the body. Then, $\mathbf{t}^{ \pm}= \pm \mathbf{P}^{ \pm} \mathbf{k}$, and Taylor expansions of $\mathbf{P}^{ \pm}$furnish

$$
\mathbf{t}^{+}+\mathbf{t}^{-}=h \mathbf{P}_{0}^{\prime} \mathbf{k}+o(h), \quad \mathbf{t}^{+}-\mathbf{t}^{-}=2 \mathbf{P}_{0} \mathbf{k}+o(h) .
$$


These are seen, with reference to (8) and (9), to be consistent with the degree of smoothness assumed for the three-dimensional deformation. Accordingly, (7) yields

$$
\operatorname{div}\left(\mathbf{P}_{0} \mathbf{1}\right)+h^{-1}\left(\mathbf{t}^{+}+\mathbf{t}^{-}\right)+h^{-1} o(h)=\mathbf{0} .
$$

From (8) and (9), it is evident that the deformation will remain bounded in the limit of small $h$ provided that

$$
\mathbf{t}^{+}+\mathbf{t}^{-}=h \mathbf{p}+o(h) \text { and } \mathbf{t}^{+}-\mathbf{t}^{-}=2 \mathbf{q}+o(1),
$$

where $\mathbf{p}$ and $\mathbf{q}$ are of order unity in magnitude. These are fields defined on $\Omega$. The equations of membrane theory emerge in the limit of small thickness. Passing to the limit $h \rightarrow 0$, we derive

$$
\operatorname{div}\left(\mathbf{P}_{0} \mathbf{1}\right)+\mathbf{p}=\mathbf{0} \quad \text { and } \quad \mathbf{P}_{0} \mathbf{k}=\mathbf{q}
$$

The second of these is seen, with the aid of $(8)$ and $(10)_{1}$, to furnish an algebraic relationship among $\nabla \mathbf{r}, \mathbf{d}$ and $\mathbf{q}$. From the work of Hilgers and Pipkin [5], this is known to be solvable uniquely for $\mathbf{d}$ whenever the strong-ellipticity condition (2) is satisfied (see also $[4,6]$ ). We write

$$
\mathbf{d}=\overline{\mathbf{d}}(\nabla \mathbf{r}, \mathbf{q}) .
$$

Equation $(14)_{1}$, with

$$
\mathbf{P}_{0}=\hat{\mathbf{P}}(\nabla \mathbf{r}+\overline{\mathbf{d}} \otimes \mathbf{k}),
$$

then furnishes a second-order differential equation for the deformation $\mathbf{r}$ of $\Omega$.

Standard mixed traction/position problems consist in the specification of $\mathbf{r}$ and the traction

$$
\tau=\mathbf{P}_{0} 1 \nu
$$

on complementary parts of the boundary curve $\partial \Omega$, with unit normal $\boldsymbol{\nu}$ exterior to $\Omega$. Here $\boldsymbol{\tau}$ is the value on $\partial \Omega$ of the exact traction field acting on a part of the cylindrical generating surface of the body where tractions are assigned.

An a posteriori restriction on $\mathbf{g}$, valid to leading order, is given by combining (9), (10) with (11) and $(13)_{1}$. Thus,

$$
\mathbf{A g}=\mathbf{p}-\{\mathcal{M}(\nabla \mathbf{r}+\overline{\mathbf{d}} \otimes \mathbf{k})[\nabla \overline{\mathbf{d}}]\} \mathbf{k},
$$

where $\mathbf{A}$ is the acoustic tensor defined by

$$
\mathbf{A} \mathbf{v}=\{\mathcal{M}(\nabla \mathbf{r}+\overline{\mathbf{d}} \otimes \mathbf{k})[\mathbf{v} \otimes \mathbf{k}]\} \mathbf{k}
$$

for all $\mathbf{v}$. This is positive definite by virtue of (2). Accordingly, (18) yields a unique field $\mathbf{g}$ for any solution $\mathbf{r}$ to the boundary-value problem for the midsurface position field.

\section{Examples}

Two cases arise in applications: 
(i) The tractions $\mathbf{t}^{ \pm}$are of order $h$.

In this case we have $\mathbf{q}=\mathbf{0}$. Thus, to leading order, the midsurface $\Omega$ is in a state of plane stress (cf. $\left.(14)_{2}\right)$, and $\mathbf{d}=\overline{\mathbf{d}}(\nabla \mathbf{r}, \mathbf{0})$. If $\Omega$ is a plane of reflection symmetry of the material properties, then the classical result $\mathbf{d}=\lambda \mathbf{n}$ follows [5], where $\lambda$ is the thickness distension and $\mathbf{n}$ is the unit normal to the current surface $\omega$. This is the image of $\Omega$ under the deformation $\mathbf{r}$.

This class of problems is exemplified by inflation, unilateral contact and pure edge loading. In the first case the tractions are given by

$$
\mathbf{t}^{ \pm}=\mp\left(p^{ \pm}\right)\left(\mathbf{F}^{*}\right)^{ \pm} \mathbf{k}, \quad \text { with } \quad p^{ \pm}=h P^{ \pm}+o(h)
$$

where $\mathbf{F}^{*}$ is the cofactor of $\mathbf{F}, p^{ \pm}$are the pressures exerted on the lateral surfaces, and $P^{ \pm}$are of order unity. We obtain

$$
\mathbf{p}=\alpha(\Delta P) \mathbf{n}
$$

where $\Delta P=P^{-}-P^{+}$is the net lateral pressure across the membrane and $\alpha$ is the areal dilation, having used Nanson's formula in the form

$$
\alpha \mathbf{n}=\mathbf{F}_{0}^{*} \mathbf{k}
$$

This class also includes unilateral inflation and contact problems for which either $\mathbf{t}^{+}$or $\mathbf{t}^{-}$vanishes, as $(13)_{1}$ and $(13)_{2}$ are then reconciled only if $\mathbf{q}=\mathbf{0}$.

(ii) The difference of the tractions is of order unity.

Equations $(13)_{1,2}$ furnish

$$
\mathbf{t}^{+}=\mathbf{q}+o(1) \quad \text { and } \quad \mathbf{t}^{-}=-\mathbf{q}+o(1)
$$

so that $\mathbf{t}^{-} \rightarrow-\mathbf{t}^{+}$as $h \rightarrow 0$. An example is furnished by a thin interphase bonded to rigid bodies at its upper and lower lateral surfaces. If the positions $\mathbf{r}^{ \pm}$there are known, then, to leading order,

$$
\mathbf{r}=\frac{1}{2}\left(\mathbf{r}^{+}+\mathbf{r}^{-}\right) \quad \text { and } \quad \mathbf{d}=h^{-1}\left(\mathbf{r}^{+}-\mathbf{r}^{-}\right)
$$

Equations (14) then furnish $\mathbf{p}$ and $\mathbf{q}$.

\section{References}

1. T.J. Healey and P. Rosakis, 1997, Unbounded branches of classical injective solutions to the forced displacement problem in nonlinear elastostatics. J. Elasticity 49, 65-78.

2. P.G. Ciarlet, 2005, An introduction to differential geometry with applications to elasticity. J. Elasticity 78-79, 3-201.

3. H. Le Dret and A. Raoult, 1996, The membrane shell model in nonlinear elasticity: A variational asymptotic derivation. J. Nonlinear Sci. 6, 59-84. 
4. D.J. Steigmann, 2007, Thin-plate theory for large elastic deformations. Int. J. Non-linear Mech. 42, 233-240.

5. M.G. Hilgers and A.C. Pipkin, 1996, Bending energy of highly elastic membranes II. Quart. Appl. Math. 54, 307-316.

6. D.J. Steigmann, 'Applications of Polyconvexity and Strong Ellipticity to Nonlinear Elasticity and Elastic Plate Theory', in: CISM Course on Applications of Poly-, Quasi-, and Rank-One Convexity in Applied Mechanics. Udine, Italy. Sept. 24-28, 2007 (to appear). 